\title{
Clinicopathological differences in radiation-induced organizing hematomas of the brain based on type of radiation treatment and primary lesions
}

\author{
Myung Sun Kim ${ }^{1}$, Se Hoon Kim ${ }^{1}$, Jong-Hee Chang ${ }^{2}$, Mina Park ${ }^{3}$, Yoon Jin Cha ${ }^{1}$ \\ Departments of ${ }^{1}$ Pathology and ${ }^{2}$ Neurosurgery, Yonsei University College of Medicine, Seoul; \\ ${ }^{3}$ Department of Radiology, Gangnam Severance Hospital, Yonsei University College of Medicine, Seoul, Korea
}

\begin{abstract}
Background: Radiation-induced organizing hematoma (RIOH) is a sporadic form of cavernous hemangioma ( $\mathrm{CH})$ that occurs after cerebral radiation. $\mathrm{RIOH}$ lesions are distinct histologically from de novo $\mathrm{CH}$; however, detailed research on this subject is lacking. In the present study, the clinical and histological features of RIOHs were evaluated based on causative lesions. Methods: The present study included 37 RIOHs confirmed by surgical excision from January 2009, to May 2020, in Yonsei Severance Hospital. All cases were divided into subgroups based on type of radiation treatment (gamma knife surgery [GKS], $n=24$ vs. conventional radiation therapy [RT], $n=$ 13) and pathology of the original lesion (arteriovenous malformation, $n=14$; glioma, $n=12$; metastasis, $n=4$; other tumors, $n=7$ ). The clinicopathological results were compared between the groups. Results: Clinical data of multiplicity, latency, and size and wall thickness of the original tumors and RIOHs were analyzed. The GKS group showed shorter latency $(5.85 \pm 4.06$ years vs. $11.15 \pm 8.27$ years, $p=$ $.046)$ and thicker tumor wall $(693.7 \pm 565.7 \mu \mathrm{m}$ vs. $406.9 \pm 519.7 \mu \mathrm{m}, \mathrm{p}=.049)$ than the conventional RT group. Significant difference was not found based on original pathology. Conclusions: $\mathrm{RIOH}$ is more likely to occur earlier with thick tumor wall in subjects who underwent GKS than in patients who underwent conventional RT. These results indicate the clinical course of RIOH differs based on type of treatment and might help determine the duration of follow-up.
\end{abstract}

Key Words: Radiation-induced organizing hematoma; Cavernous hemangioma; Gamma knife surgery; Radiation therapy; Latency

Received: June 28, 2021 Revised: August 24, 2021 Accepted: August 30, 2021

Corresponding Author: Yoon Jin Cha, MD, PhD, Department of Pathology, Gangnam Severance Hospital, Yonsei University College of Medicine, 211 Eonju-ro, Gangnam-gu, Seoul 06273, Korea

Tel: +82-2-2019-3540, Fax: +82-2-3463-2103, E-mail: yooncha@yuhs.ac

Radiation-indicated cavernous hemangioma (RICH) refers to a localized vascular, tumor-like lesion that develops after cerebral radiation [1-3]. The lesion occurs mainly in children and young people, with variable latency periods after radiation treatment and diverse original lesions, including arteriovenous malformation (AVM), glioma, and metastatic tumor [4,5]. Similar to de novo cavernous hemangioma (CH), RICH appears as an enhancing lesion with popcorn-like appearance and partial hemosiderin rim on magnetic resonance imaging (MRI) and is observed histologically as a vascular-rich hemorrhagic lesion [6-8]. Thus, $\mathrm{RICH}$ has been regarded as a sporadic form of de novo $\mathrm{CH}$ that appears as a late complication of cerebral radiation $[9,10]$.

Recently, in several studies in which RICH was compared with de novo $\mathrm{CH}$, RICH was shown a potentially distinct group of diseases with different pathogenesis compared with previously known $\mathrm{CHs}$ [6,11-13]. Previous research has reported that RICH following stereotactic radiosurgery shows some distinguishing features on MRI such as an unilocular cystic area with some solid component and prominent perilesional edema, and de novo $\mathrm{CH}$ appears to have a complete hemosiderin rim and less prominent perilesional edema [6]. Furthermore, RICH is more likely to be an inactive organizing hematoma rather than a vascular malformation. Therefore, the term "radiation-induced organizing hematoma (RIOH)" was proposed to describe the lesions more appropriately and replace the term $\mathrm{RICH}$, which might be a misnomer for these lesions [6]. All the authors of the current study agreed to this concept, and the term RIOH, instead of RICH, was used throughout the manuscript. 
Detailed studies on $\mathrm{RIOH}$ are limited. In particular, $\mathrm{RIOH}$ lesions have been reported irrespective of radiation dose, type of malignancy, or radiation type, such as gamma knife surgery (GKS) or conventional radiation therapy (RT) [11]. However, studies to improve the understanding of $\mathrm{RIOH}$ lesions have not been conducted. In the present study, RIOH lesions were better defined, and their clinicopathological characteristics, including original pathology and type of radiation treatment, were compared.

\section{MATERIALS AND METHODS}

\section{Patient selection and clinicopathological examination}

Between January 2009 and May 2020, a total of 37 cases of pathologically confirmed RIOH was selected from Severance Hospital. For each case, the size of both the original tumor and $\mathrm{RIOH}$ was obtained. The size of original tumor was measured from preoperative imaging, and the size of $\mathrm{RIOH}$ were obtained through microscopic examination. The difference between the maximal diameter of primary tumor and $\mathrm{RIOH}$ was measured. All the pathologic slides were reviewed, and the tumor wall thickness of RIOH was measured at the thickest point of the submitted tissue under light microscope.

All medical records were reviewed, and clinical data including age at the time of $\mathrm{RIOH}$ detection, sex, radiologic findings, locations of the original tumor and $\mathrm{RIOH}$, multiplicity, and latency period were compared between the original tumor and RIOH. A radiologist (M.P.) reviewed all MRI scans of patients, focusing on differences in radiologic findings including perilesional edema and hemosiderin rim depending on type of RT. Furthermore, information on previous treatments was collected. Cases were classified into two groups based on previous type of radiation (GKS, $n=24$ vs. conventional $\mathrm{RT}, \mathrm{n}=13$ ) and into four groups based on preceding pathology of the original tumor (AVM, $\mathrm{n}=14$; glioma, $\mathrm{n}=12$; metastasis, $\mathrm{n}=4$; other tumors, $\mathrm{n}=7$ ). The clinicopathological parameters were analyzed. For patients who had been treated multiple times or who underwent both GKS and RT, the groups and latency were divided and calculated based on timing and method of the last treatment.

\section{Statistical analysis}

Statistical analyses were performed using SPSS ver. 21.0 (IBM Corp., Armonk, NY, USA). Continuous and categorical variables were analyzed using the non-parametric Mann-Whitney U test and the chi-square test, respectively, and $\mathrm{p}<.05$ was considered statistically significant.

\section{RESULTS}

\section{Patient characteristics}

A total of 37 samples was included in the study. Baseline patient characteristics are summarized in Table 1 . RIOH samples were from 14 males $(37.8 \%)$ and 23 females $(62.2 \%)$. Twenty-four patients $(64.9 \%)$ underwent GKS, and $13(35.1 \%)$ were treated with conventional RT. Original tumor pathologies were as follows: $\operatorname{AVM}(\mathrm{n}=14,37.8 \%)$, brain tumors (11 gliomas and 1 ependymoma; $n=12,32.4 \%)$, metastasis $(n=4,10.8 \%)$, and other tumors ( 3 schwannomas, two nasopharyngeal cancers, one pituitary tumor, and one craniopharyngioma; $\mathrm{n}=7,18.9 \%$ ). The cases of nasopharyngeal cancer were subcategorized into 'other tumors' in this study because postoperative RT often is used for nasopharyngeal cancer, and the central nervous system area usually is included in the radiation field.

\section{Clinicopathological differences between $\mathrm{RIOH}$ lesions}

The overall results of the clinical and pathological comparisons are summarized in Tables 2 and 3. RIOH cases were divided into two groups based on previous type of radiation (GKS or conventional RT). Histologically, RIOH shows a hematoma-like area with a reduced number of hyalinized thin-walled vessels with fibrin and infiltrating foamy macrophages in the vessel walls. Conversely, de novo $\mathrm{CH}$ shows a thick, hyalinized wall without prominent macrophage infiltration (Fig. 1). The results

Table 1. Baseline characteristics of the patients $(n=37)$

\begin{tabular}{lr}
\hline Characteristic & No. (\%) \\
\hline Age at the time of RIOH detection (yr), mean \pm SD & $46.57 \pm 13.79$ \\
Sex & \\
Male & $14(37.8)$ \\
Female & $23(62.2)$ \\
Original pathology & \\
AVM & $14(37.8)$ \\
Brain tumor & $12(32.4)$ \\
Metastasis & $4(10.8)$ \\
Other tumors & $7(19.0)$ \\
Type of treatment & \\
GKS & $24(64.9)$ \\
RTx & $13(35.1)$ \\
Tumor location & \\
Frontal lobe & $10(27.0)$ \\
Parietal lobe & $5(13.5)$ \\
Temporal lobe & $6(16.2)$ \\
Occipital lobe & $4(10.8)$ \\
Other (including sellar lesion) & $12(32.5)$ \\
\hline
\end{tabular}

$\mathrm{RIOH}$, radiation-induced organizing hematoma; SD, standard deviation; AVM, arteriovenous malformation; GKS, gamma knife surgery; RTx, radiation therapy. 
Table 2. Clinicopathological differences between 37 radiation-induced organizing hematomas

\begin{tabular}{|c|c|c|c|c|c|c|c|c|}
\hline \multirow{2}{*}{ Variable } & \multicolumn{2}{|c|}{ Radiation treatment } & \multirow{2}{*}{$p$-value } & \multicolumn{4}{|c|}{ Original pathology } & \multirow{2}{*}{$p$-value } \\
\hline & GKS $(n=24)$ & $\operatorname{RTx}(n=13)$ & & $\operatorname{AVM}(n=14)$ & Brain tumor $(n=12)$ & Metastasis $(n=4)$ & Others $(n=7)$ & \\
\hline \multicolumn{9}{|l|}{ Tumor size (cm) } \\
\hline Primary tumor & $3.35 \pm 1.22$ & $3.04 \pm 1.08$ & .387 & $3.87 \pm 1.26$ & $2.85 \pm 1.04$ & $2.92 \pm 0.60$ & $2.85 \pm 1.05$ & .081 \\
\hline $\mathrm{RIOH}$ & $2.01 \pm 0.80$ & $1.51 \pm 0.55$ & .055 & $2.06 \pm 0.82$ & $1.50 \pm 0.68$ & $2.20 \pm 0.41$ & $1.75 \pm 0.75$ & .459 \\
\hline (Size differences) & $1.34 \pm 1.33$ & $1.53 \pm 1.28$ & .695 & $1.81 \pm 1.49$ & $1.35 \pm 1.25$ & $0.72 \pm 0.45$ & $1.10 \pm 1.23$ & .425 \\
\hline Tumor wall thickness ( $\mu \mathrm{m})$ & $693.7 \pm 565.7$ & $406.9 \pm 519.7$ & .049 & $714.2 \pm 610.9$ & $560.0 \pm 630.6$ & $362.5 \pm 47.8$ & $538.5 \pm 518.0$ & .752 \\
\hline Latency (yr) & $5.85 \pm 4.06$ & $11.15 \pm 8.27$ & .046 & $8.92 \pm 7.01$ & $7.55 \pm 5.06$ & $2.28 \pm 3.45$ & $8.68 \pm 7.28$ & .161 \\
\hline
\end{tabular}

Values are presented as mean \pm SD.

GKS, gamma knife surgery; RTx, radiation therapy; AVM, arteriovenous malformation; RIOH, radiation-induced organizing hematoma; SD, standard deviation.

Table 3. Correlation between multiplicity and treatment/original pathology

\begin{tabular}{lcccc}
\hline & \multicolumn{4}{c}{ Multiplicity } \\
\cline { 2 - 4 } & Total & No & Yes & p-value \\
\hline GKS & 24 & $21(87.5)$ & $3(12.5)$ & .586 \\
RTx & 13 & $11(84.6)$ & $2(15.4)$ & \\
AVM & 14 & $13(92.8)$ & $1(7.2)$ & .155 \\
Brain tumor & 12 & $10(83.3)$ & $2(16.7)$ & \\
Metastasis & 4 & $2(50.0)$ & $2(50.0)$ & \\
Others & 7 & $7(100)$ & 0 & \\
\hline
\end{tabular}

GKS, gamma knife surgery; RTx, radiation therapy; AVM, arteriovenous malformation.

showed significantly shorter latency in the GKS group than in the conventional RT group $(5.85 \pm 4.06$ years vs. $11.15 \pm 8.27$ years, $\mathrm{p}=.046)$. In addition, the RIOH in the GKS group had significantly thicker tumor wall than that in the conventional RT group $(693.7 \pm 565.7 \mu \mathrm{m}$ vs. $406.9 \pm 519.7 \mu \mathrm{m}, \mathrm{p}=.049)$ (Fig. 2). Significant differences were not observed in age, tumor size of primary tumor, or RIOH. The primary tumor size did not differ between groups. However, the size of RIOH lesions tended to be larger in the GKS group than in the conventional RT group $(\mathrm{p}=.055)$. The multiplicity of RIOH was not significantly different between the groups. In terms of original pathology, the four groups based on preceding pathology were compared, and no significant differences were found in any of the clinicopathological parameters.

The results of the radiologic findings are summarized in Table 4. All patients with GKS-induced RIOH and 11 patients with RT-induced RIOH showed perilesional edema on T2weighted images. Seven patients in the GKS-induced RIOH group showed subacute stage hemorrhage on T1-weighted images, and 21 showed hemosiderin rim deposit on T2-weighted images. Similarly, nine patients in the RT-induced RIOH group showed subacute stage hemorrhage, and 11 cases showed hemosiderin rim. Significant difference in radiologic image findings including perilesional edema $(\mathrm{p}=.223)$, subacute stage hemor- rhage $(\mathrm{p}=.059)$ and hemosiderin deposit in MR image $(\mathrm{p}=$ .679), was not observed between the GKS and RTx groups.

No significant differences were found in other parameters. In the GKS group, the mean age at the time of RIOH diagnosis was 46.9 years; the mean sizes of primary tumor and $\mathrm{RIOH}$ were 3.35 $\mathrm{cm}$ and $2.01 \mathrm{~cm}$, respectively; and size difference between primary tumor and $\mathrm{RIOH}$ was $1.34 \mathrm{~cm}$. In the conventional RT group, the mean age was 45.8 years; the sizes of primary tumor and RIOH measured $3.04 \mathrm{~cm}$ and $1.51 \mathrm{~cm}$ on average, respectively; and the size difference between primary tumor and RIOH was $1.53 \mathrm{~cm}$. However, the size of RIOH lesions tended to be larger in the GKS group than in the conventional RT group $(\mathrm{p}=.055)$.

In terms of original pathology, the four groups based on preceding pathology were compared. The mean age in the AVM, brain tumor, metastasis, and other tumors groups was 43.1 years, 43.5 years, 57.2 years, and 52.7 years, respectively. The mean sizes of the primary tumor and RIOH were $3.87 \mathrm{~cm}$ and $2.06 \mathrm{~cm}$ in the AVM group, $2.85 \mathrm{~cm}$ and $1.50 \mathrm{~cm}$ in the brain tumor group, $2.92 \mathrm{~cm}$ and $2.20 \mathrm{~cm}$ in the metastasis group, and $2.85 \mathrm{~cm}$ and $1.75 \mathrm{~cm}$ in the other tumors group, respectively. The size difference was $1.81 \mathrm{~cm}, 1.35 \mathrm{~cm}, 0.72 \mathrm{~cm}$, and $1.10 \mathrm{~cm}$ in the AVM, brain tumor, metastasis, and other tumors groups, respectively. The mean tumor wall thickness was 714.2 $\mu \mathrm{m}$ in the AVM group, $560.0 \mu \mathrm{m}$ in the brain tumor group, $362.5 \mu \mathrm{m}$ in the metastasis group, and $538.5 \mu \mathrm{m}$ in the other tumors group. The average latency in the AVM, brain tumor, metastasis, and other tumors groups was 8.92 years, 7.55 years, 2.28 years, and 8.68 years, respectively. No significant difference was found in any of the clinicopathological parameters.

Three patients in the GKS group showed multiplicity, two in the conventional RT group, 1 in the AVM group, 2 in the brain tumor group, and two in the metastasis group; however, statistical difference was not found between the groups. 

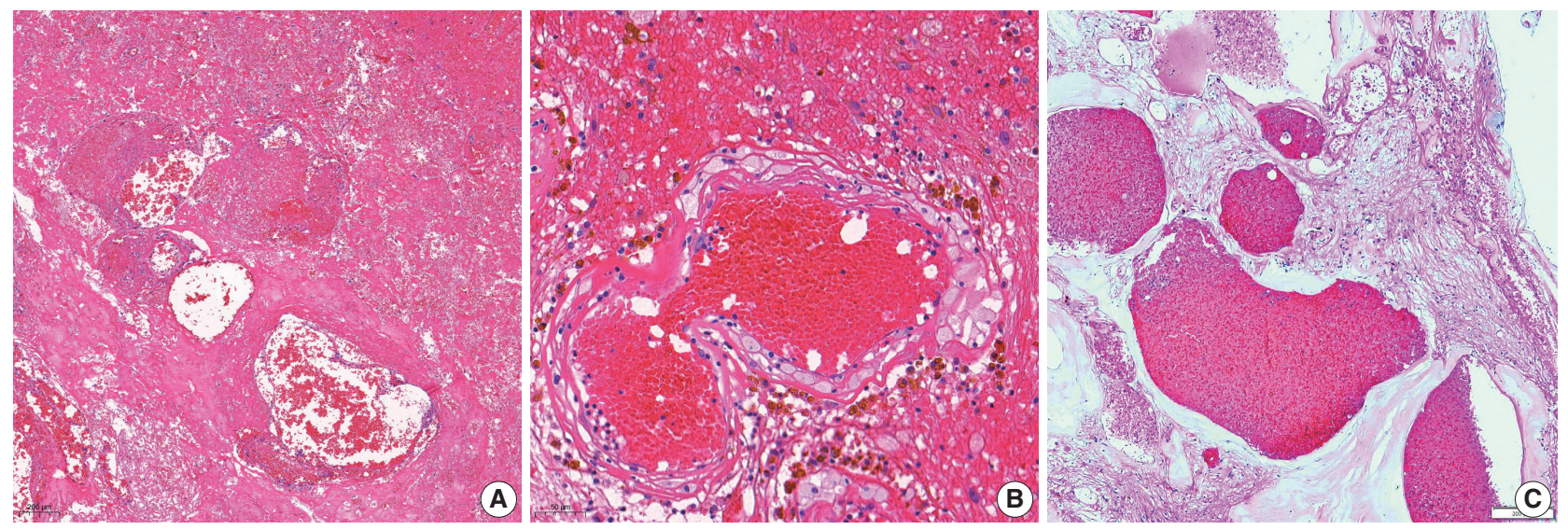

Fig. 1. The histology of radiation-induced organizing hematoma $(\mathrm{RIOH})$ and de novo cavernous hemangioma $(\mathrm{CH})$; $\mathrm{Microscopically,} \mathrm{RIOH}$ shows a hematoma-like area composed of hyalinized vessels with fibrin and infiltrating foamy macrophages ( $\mathrm{A}, \mathrm{B})$, and de novo $\mathrm{CH}$ consists of clusters of well-formed vascular lumens (C).

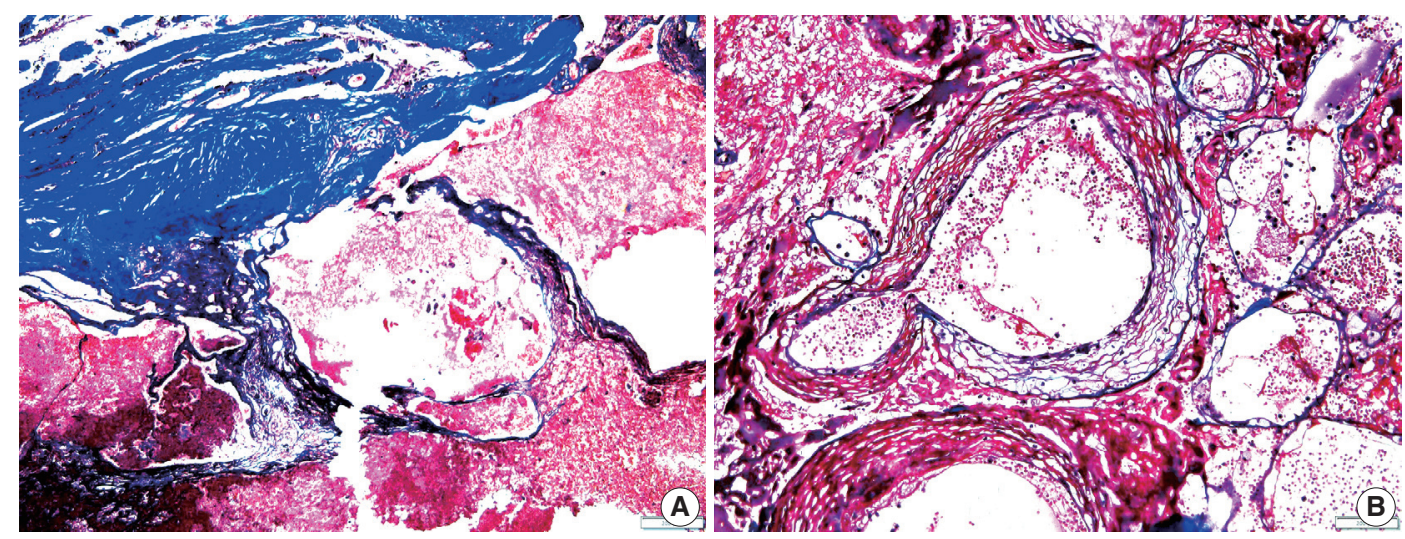

Fig. 2. Gamma knife surgery-induced radiation-induced cavernous hemangioma $(\mathrm{RIOH})$ shows relatively thicker tumor walls (A) compared with conventional radiation therapy-induced $\mathrm{RIOH}(\mathrm{B})$ (Masson's trichrome).

Table 4. Radiological differences between GKS-induced and conventional RTx-induced RIOH

\begin{tabular}{|c|c|c|c|c|c|c|c|c|c|c|}
\hline & \multirow[b]{2}{*}{ Total } & \multicolumn{2}{|c|}{ Perilesional edema } & \multirow[t]{2}{*}{$p$-value } & \multicolumn{2}{|c|}{ Subacute stage hemorrhage ( $\mathrm{T} 1$ high) } & \multirow[t]{2}{*}{$p$-value } & \multicolumn{2}{|c|}{$\begin{array}{l}\text { Hemosiderin deposit } \\
\text { (T2 dark rim) }\end{array}$} & \multirow[t]{2}{*}{$p$-value } \\
\hline & & Absent & Present & & Absent & Present & & Absent & Present & \\
\hline GKS & 24 & 0 & $23(95.8)^{a}$ & .223 & $9(37.5)$ & $7(29.1)^{b}$ & .059 & $2(8.3)$ & $21(87.5)^{\mathrm{a}}$ & .679 \\
\hline $\mathrm{RTx}$ & 13 & $2(15.3)$ & $11(84.6)$ & & $4(30.7)$ & $9(69.2)$ & & $2(15.3)$ & $11(84.6)$ & \\
\hline
\end{tabular}

GKS, gamma knife surgery; RTx, radiation therapy; RIOH; radiation-induced organizing hematoma; MRI, magnetic resonance imaging.

ane case was undetectable on MRI; 'Eight cases were excluded that did not include the T1 series in preoperative MRI.

\section{DISCUSSION}

$\mathrm{RIOH}$ has been considered a sporadic form of de novo $\mathrm{CH}$ occurring as a late complication of cerebral radiation. $\mathrm{RIOH}$ occurs in children mainly after the use of cerebral radiation to treat medulloblastoma, glioma, or AVM or for prophylactic treatment of hematological malignancies such as acute lymphoblastic leukemia $[4,5,14,15]$. However, RIOH is rare in adults
[14,16]. In a previous study with 84 cases of RIOH, the average age at diagnosis was 20.6 years, the median was 17 years, and the average latency to development of RIOH was 10.3 years, with a median of 8 years [11]. One rare case of RIOH has been diagnosed at 52 years after RT [17].

The pathophysiology of RIOH is not well-known; however, two hypotheses have been suggested: occult $\mathrm{CHs}$ that were previously present respond to radiation and become apparent; de 
novo formation of the lesion in response to radiation [10]. The de novo mechanisms might include a series of processes, such as vascular injury, proliferation of the vascular wall, necrosis, and ischemia due to narrowing of the lumen. Previous studies showed increased vascular endothelial growth factor after exposure to radiation in rats, supporting this hypothesis [18].

Patients with de novo $\mathrm{CH}$ can be treated with antiepileptic drugs and regular follow-up but are usually recommended to undergo surgery if possible in case the symptoms worsen or the size changes due to the risk of bleeding. In RIOH, which is regarded a sporadic form of de novo $\mathrm{CM}$, surgical treatment is considered the standard treatment option following the treatment algorithm for de novo CM. However, if RIOH is considered a separate disease entity with different pathogenesis and clinical course than de novo $\mathrm{CM}$, conservative treatment modalities could be considered a in addition to invasive surgical treatments, which could cause neurological side effects. Therefore, several studies have been conducted to compare the pathogenesis of RIOH and de novo $\mathrm{CH}$, and several notable results were reported. Compared with de novo $\mathrm{CH}$, $\mathrm{RIOH}$ was found to develop at younger age, symptoms at the time of diagnosis were milder, and tended to be more multifocal. However, the prevalence of a hemorrhagic event, the most fatal complication, was not significantly different [11-13].

Considering the hematoma-like area and infiltration of foamy macrophage, RIOH appears more likely to be an inactive hemangioma-like lesion, which might be closer to a recanalized cavitary hematoma induced by high-dose radiation than to vascular malformation [6]. Therefore, we suggest the use of the term $\mathrm{RIOH}$ rather than RICH.

In the present study, we hypothesized that RIOH would show clinical and histological differences depending on treatment type or primary pathology. Based on the treatment type, the GKS group showed shorter latency. In previous studies, several factors affecting the duration of the latency period have been reported, including radiation dose $>30 \mathrm{~Gy}$ and RT before 10 years of age $[11,14,15,19]$, with increased risk of hemorrhagic event [9]. Hypothetically, the shorter latency observed in the GKS group might be due to its requirement of a higher dose in a smaller target area compared with conventional RT or focused damage that could accelerate tissue necrosis and tumorigenesis.

The present study had several limitations. First, the small sample size might have led to skewed statistical results. Second, although all available clinical, radiologic, and pathologic data were collected, some parameters might be inconsistent in retrospec- tive analysis. Third, the subjects were older in the present study. As previously stated, $\mathrm{RIOH}$ occurs primarily in younger patients, however, in the present study, the mean age of the subjects was 46.6 years, which is an older age group compared with other reports (median 31.1 years [12]; mean age at the time of radiation 10.4 years with mean latency of 10.3 years [11]). Histopathological differences can exist in the RIOH of older subjects compared with younger subjects and could affect the results. However, only 37 patients were included in this study, most of whom were in their 30s or older, explaining why the results differ from those of previous studies. Further research with a larger cohort is needed to verify the results.

We hypothesized that the size of RIOH after conventional RT would be larger than after GKS because conventional RT is applied for larger lesions. However, the average size of the lesion tended to be larger in the GKS group, although the difference was not statistically significant. Furthermore, we hypothesized that the size difference between primary tumor and RIOH would be greater in the GKS group than in the RT group; however, significant difference was not observed. The repeated treatment applied in the same lesion, the error in the measurement of the radiological/pathological size, and the limitations of the present study described above might have affected these results.

In summary, RIOH after GKS tended to occur earlier and had thinner tumor wall than RIOH after conventional RT. The original pathology of RIOH had no effect on the histologic and clinical features of RIOH. These results suggest that the clinical course of RIOH differs based on type of treatment. Understanding the unique pathophysiology of RIOH, one of the most wellknown complications of cerebral radiation, has become more important as the survival rate of brain tumor patients increases. Therefore, further investigation in the form of prospective studies with larger cohorts is required to elucidate more detailed clinical and histologic features of patients to provide appropriate medical management and predict the clinical course more accurately.

\section{Ethics Statement}

This retrospective study was approved by the Institutional Review Board of Severance Hospital (4-2020-0186), and patient informed consent was waived. All procedures were performed in accordance with the 1964 Declaration of Helsinki and its later amendments or comparable ethical standards.

\section{Availability of Data and Material}

All data generated or analyzed during the study are included in this published article (and its supplementary information files).

\section{Code Availability}

Not applicable. 


\section{ORCID}

Myung Sun Kim https://orcid.org/0000-0003-1521-2586

Se Hoon Kim https://orcid.org/0000-0001-7516-7372

Jong-Hee Chang https://orcid.org/0000-0003-1509-9800

Mina Park https://orcid.org/0000-0002-2005-7560

Yoon Jin Cha https://orcid.org/0000-0002-5967-4064

\section{Author Contributions}

Conceptualization: YJC, SHK. Data curation and interpretation: MSK, YJC, MP, SHK. Supervision: SHK, JHC, YJC. Writing-original draft: MSK, YJC. Writing-review \& editing: YJC, JHC, SHK. Approval of final manuscript: all authors.

\section{Conflicts of Interest}

S.H.K., a contributing editor of the Journal of Pathology and Translational Medicine, was not involved in the editorial evaluation or decision to publish this article. All remaining authors have declared no conflicts of interest.

\section{Funding Statement}

No funding to declare.

\section{References}

1. Heckl S, Aschoff A, Kunze S. Radiation-induced cavernous hemangiomas of the brain: a late effect predominantly in children. Cancer 2002; 94: 3285-91.

2. Park YS, Kim SH, Chang JH, Chang JW, Park YG. Radiosurgery for radiosurgery-induced cavernous malformation. World Neurosurg 2011; 75: 94-8.

3. Vinchon M, Leblond P, Caron S, Delestret I, Baroncini M, Coche B. Radiation-induced tumors in children irradiated for brain tumor: a longitudinal study. Childs Nerv Syst 2011; 27: 445-53.

4. Humpl T, Bruhl K, Bohl J, Schwarz M, Stoeter P, Gutjahr P. Cerebral haemorrhage in long-term survivors of childhood acute lymphoblastic leukaemia. Eur J Pediatr 1997; 156: 367-70.

5. Larson JJ, Ball WS, Bove KE, Crone KR, Tew JM, Jr. Formation of intracerebral cavernous malformations after radiation treatment for central nervous system neoplasia in children. J Neurosurg 1998; 88: 51-6.

6. Cha YJ, Nahm JH, Ko JE, et al. Pathological Evaluation of Radiation-Induced Vascular Lesions of the Brain: Distinct from De Novo Cavernous Hemangioma. Yonsei Med J 2015; 56: 1714-20.

7. Hegde AN, Mohan S, Lim CC. CNS cavernous haemangioma: "popcorn" in the brain and spinal cord. Clin Radiol 2012; 67: 380-8.

8. Mann P, Kleinschmidt-DeMasters BK. CNS Masson Tumors: Frequent Association With Therapeutic Radiation. Am J Surg Pathol 2016; 40: 81-93.

9. Koike T, Yanagimachi N, Ishiguro $\mathrm{H}$, et al. High incidence of radiation-induced cavernous hemangioma in long-term survivors who underwent hematopoietic stem cell transplantation with radiation therapy during childhood or adolescence. Biol Blood Marrow Transplant 2012; 18: 1090-8.

10. Nimjee SM, Powers CJ, Bulsara KR. Review of the literature on de novo formation of cavernous malformations of the central nervous system after radiation therapy. Neurosurg Focus 2006; 21: e4.

11. Keezer MR, Del Maestro R. Radiation-induced cavernous hemangiomas: case report and literature review. Can J Neurol Sci 2009; 36: 303-10.

12. Cutsforth-Gregory JK, Lanzino G, Link MJ, Brown RD, Jr., Flemming KD. Characterization of radiation-induced cavernous malformations and comparison with a nonradiation cavernous malformation cohort. J Neurosurg 2015; 122: 1214-22.

13. Duhem R, Vinchon M, Leblond P, Soto-Ares G, Dhellemmes P. Cavernous malformations after cerebral irradiation during childhood: report of nine cases. Childs Nerv Syst 2005; 21: 922-5.

14. Furuse M, Miyatake SI, Kuroiwa T. Cavernous malformation after radiation therapy for astrocytoma in adult patients: report of 2 cases. Acta Neurochir (Wien) 2005; 147: 1097-101.

15. Mariniello G, De Liso M, Russo C, et al. Radiation-induced brain cavernomas in elderly: review of the literature and a rare case report. Acta Biomed 2019; 90: 77-83.

16. Sasagawa Y, Akai T, Itou S, Iizuka H. Gamma knife radiosurgeryinduced cavernous hemangioma: case report. Neurosurgery 2009; 64: E1006-7.

17. Bejjani GK, Caputy AJ, Kurtzke RN, Duong DH, Sekhar LN. Remote hemorrhage of a pontine cavernous angioma fifty-two years after cerebral irradiation. Acta Neurochir (Wien) 1997; 139: 583-4.

18. Tsao MN, Li YQ, Lu G, Xu Y, Wong CS. Upregulation of vascular endothelial growth factor is associated with radiation-induced blood-spinal cord barrier breakdown. J Neuropathol Exp Neurol 1999; 58: 1051-60.

19. Strenger V, Sovinz P, Lackner H, et al. Intracerebral cavernous hemangioma after cranial irradiation in childhood. Incidence and risk factors. Strahlenther Onkol 2008; 184: 276-80. 